\title{
Age-related upper limits of normal for maximum upright exercise pulmonary haemodynamics
}

\author{
Rudolf K.F. Oliveira ${ }^{1,2,3,6}$, Manyoo Agarwal ${ }^{1,2,6}$, Julie A. Tracy ${ }^{1,2}$, Abbey L. Karin ${ }^{1,2}$, \\ Alexander R. Opotowsky ${ }^{2,4,5}$, Aaron B. Waxman ${ }^{1,2}$ and David M. Systrom ${ }^{1,2}$ \\ Affiliations: 'Division of Pulmonary and Critical Care Medicine, Dept of Medicine, Brigham and Women's \\ Hospital and Harvard Medical School, Boston, MA, USA. ${ }^{2}$ Heart and Vascular Center, Brigham and Women's \\ Hospital, Boston, MA, USA. ${ }^{3}$ Division of Respiratory Diseases, Dept of Medicine, Federal University of \\ São Paulo (UNIFESP), São Paulo, SP, Brazil. 'Dept of Cardiology, Boston Children's Hospital, Boston, MA, \\ USA. ${ }^{5}$ Division of Cardiovascular Medicine, Dept of Medicine, Brigham and Women's Hospital, Boston, MA, \\ USA. ${ }^{6}$ These authors contributed equally to the study.
}

Correspondence: David M. Systrom, Pulmonary and Critical Care Medicine, Dept of Medicine, Brigham and Women's Hospital. Clinics 3, 75 Francis Street, Boston, MA 02115, USA. E-mail: dsystromapartners.org

ABSTRACT The exercise definition of pulmonary hypertension was eliminated from the pulmonary hypertension guidelines in part due to uncertainty of the upper limits of normal (ULNs) for exercise haemodynamics in subjects $>50$ years old.

The present study, therefore, evaluated the pulmonary haemodynamic responses to maximum upright incremental cycling exercise in consecutive subjects who underwent an invasive cardiopulmonary exercise testing for unexplained exertional intolerance, deemed normal based on preserved exercise capacity and normal resting supine haemodynamics. Subjects aged $>50$ years old $(n=41)$ were compared with subjects $\leqslant 50$ years old $(n=25)$. ULNs were calculated as mean +2 sD.

Peak exercise mean pulmonary arterial pressure was not different for subjects $>50$ and $\leqslant 50$ years old ( $23 \pm 5$ versus $22 \pm 4 \mathrm{mmHg}, \mathrm{p}=0.22$ ), with ULN of 33 and $30 \mathrm{mmHg}$, respectively. Peak cardiac output was lower in older subjects (median (interquartile range): 12.1 (9.4-14.2) versus $16.2(13.8-19.2) \mathrm{L} \cdot \mathrm{min}^{-1}$, $\mathrm{p}<0.001)$. Peak pulmonary vascular resistance was higher in older subjects compared with younger subjects (mean \pm SD: $1.20 \pm 0.45$ versus $0.82 \pm 0.26$ Wood units, $\mathrm{p}<0.001$ ), with ULN of 2.10 and 1.34 Wood units, respectively.

We observed that subjects $>50$ and $\leqslant 50$ years old have different pulmonary vascular responses to exercise. Older subjects have higher pulmonary vascular resistance at peak exercise, resulting in different exercise haemodynamics ULNs compared with the younger population.

$@$ ERSpublications

Normal subjects $>50$ years old have a different pulmonary vascular response to exercise compared with younger subjects http://ow.ly/UsNMT

This article has supplementary material available from erj.ersjournals.com

Received: Aug 062015 | Accepted after revision: Oct 312015 | First published online: Dec 172015

Support statement: R.K.F. Oliveira receives funds from the São Paulo Research Foundation (FAPESP), grant 2014/ 12212-5. A.B. Waxman and D.M. Systrom are funded by NIH 2R01HL060234-12A1 and U01HL125215-01. A.R. Opotowsky receives support from the Dunlevie Family Fund. Funding information for this article has been deposited with FundRef.

Conflict of interest: None declared.

Copyright @ERS 2016 


\section{Introduction}

Historically, pulmonary hypertension has been defined as a mean pulmonary artery pressure (mPAP) $\geqslant 25 \mathrm{mmHg}$ at rest supine right heart catheterisation (RHC) or a value $\geqslant 30 \mathrm{mmHg}$ during exercise [1]. However, the exercise definition was abandoned by an expert consensus at the 2008 Dana Point Fourth World Symposium on Pulmonary Hypertension [2] and this decision was maintained at the 2013 Nice Fifth World Symposium on Pulmonary Hypertension [3], in part due to uncertainty regarding the age-related upper limits of normal (ULNs) for exercise haemodynamics. However, recent studies suggest that exercise pulmonary hypertension, characterised by normal resting haemodynamics with an abnormal pulmonary vascular response to exercise, may be associated with symptoms [4,5], decreased quality of life [5], right ventricular/pulmonary vascular uncoupling [4] and impaired exercise capacity [4-6]. Additionally, several studies have suggested exercise pulmonary hypertension may be a mild, early and perhaps more treatable stage of established resting pulmonary hypertension [4-9].

Currently, it is unclear how to define the pulmonary vascular response to exercise as "abnormal" [10], especially in subjects $>50$ years old due to the lack of directly measured maximum exercise haemodynamic data in this population [3], whose pulmonary vasculature may respond differently to exercise than younger subjects [11-15], and the lack of a unified exercise test protocol. Hence, there is a need to better delineate pulmonary haemodynamic patterns during exercise as a function of age.

In the current study, therefore, we evaluated the pulmonary haemodynamic responses to maximum incremental upright cycling exercise in consecutive subjects who underwent invasive cardiopulmonary exercise testing (iCPET) for unexplained exertional intolerance, deemed normal based on a preserved exercise capacity and a normal resting supine RHC. We hypothesised that subjects $>50$ years old would present a different pulmonary vascular response to exercise compared with subjects $\leqslant 50$ years old.

\section{Methods}

\section{Design and study population}

We analysed 612 consecutive subjects referred to the Dyspnea Clinic at Brigham and Women's Hospital between January 2012 and December 2014 who underwent resting supine RHC followed by upright symptom-limited iCPET on a cycle ergometer as a part of their clinical evaluation for unexplained exertional intolerance [16].

Subjects were selected based on preserved exercise capacity during iCPET and normal resting supine haemodynamics at RHC, using the following criteria: 1) maximal oxygen uptake $\left(V^{\prime} \mathrm{O}_{2} \max \right) \geqslant 80 \%$ predicted [17], 2) maximal cardiac output $(\mathrm{COmax}) \geqslant 80 \%$ predicted $[4,18]$, 3) rest supine mPAP $\leqslant 20 \mathrm{mmHg}$ and 4) rest supine pulmonary arterial wedge pressure (PAWP) $\leqslant 12 \mathrm{mmHg}$. Subjects aged $>50$ years were compared with subjects $\leqslant 50$ years old.

Contemporary transthoracic echocardiography and spirometry tests were also analysed. Partners Human Research Committee approved this retrospective study (protocol 2011P000272).

\section{Right heart catheterisation}

Resting supine RHC was performed using a Swan-Ganz catheter (Edwards Lifesciences, Irvine, CA, USA) inserted percutaneously via the internal jugular vein under ultrasound and fluoroscopic guidance. An arterial line was placed into the radial artery using a 20-gauge intravenous catheter or 5-French sheath. Zero-pressure calibration was performed at the mid-axillary line with the patient in a supine position [19]. CO at RHC was calculated using Fick's method and an estimated oxygen consumption (130xbody surface area).

\section{Invasive cardiopulmonary exercise testing}

Maximum symptom-limited incremental iCPET was performed using an upright cycle ergometer and a breath-by-breath metabolic cart (ULTIMA CPX; Medical Graphics, St Paul, MN, USA) with subjects breathing room air. All tests were preceded by a 3-min warm-up period of unloaded cycling at 55-65 rpm. The work ramp was individually selected based on the history of exercise tolerance in the field, ranging from 10 to $25 \mathrm{~W} \mathrm{~min}^{-1}$. A maximum effort at peak exercise was confirmed by peak respiratory exchange ratio $(\mathrm{RER}) \geqslant 1.05$ and/or peak heart rate $(\mathrm{HR}) \geqslant 85 \%$ predicted.

Minute ventilation, breath-by-breath pulmonary gas exchange, HR, radial arterial blood pressure, right atrial pressure (RAP) and pulmonary arterial pressure (PAP) were continuously measured throughout the test. PAWP was obtained at rest and at the end of each minute of exercise. Upright zero-pressure calibration was performed at $5 \mathrm{~cm}$ below the axillary fold with the subject at rest and again during freewheeling exercise [20].

At rest upright, and during the last $15 \mathrm{~s}$ of each minute of exercise, systemic arterial and mixed venous blood samples were simultaneously collected from the radial artery and distal pulmonary artery, respectively. 
By co-oximetry, oxygen saturation, haemoglobin concentration, and arterial and mixed venous oxygen content were measure for each blood sample. $\mathrm{CO}$ was then calculated by the Fick principle using a simultaneously measured $V^{\prime} \mathrm{O}_{2}$. Predicted COmax was calculated from predicted $V^{\prime} \mathrm{O}_{2} \max$ and an assumed maximal arterial-mixed venous oxygen content difference equivalent to a normal haemoglobin concentration of $14 \mathrm{~g} \cdot \mathrm{dL}^{-1}$ for healthy subjects $[18,21]$.

\section{Haemodynamics and pressure waveform analysis}

Pressure waveforms were recorded and reviewed using the same haemodynamic monitoring system (Xper Cardio Physiomonitoring System; Philips, Melborne, FL, USA). RAP, PAP and PAWP waveforms at rest supine, rest upright and peak exercise were individually reviewed. Pressure values were obtained by averaging the waveforms over at least three full respiratory cycles [20, 22].

Total pulmonary resistance (TPR) was calculated as the ratio of mPAP to the CO and expressed as Wood units (WU). Pulmonary vascular resistance (PVR) was calculated as the ratio of the transpulmonary gradient (TPG, i.e. difference between $\mathrm{mPAP}$ and PAWP) to the CO and expressed as WU. Pulmonary vascular compliance (PVC) was calculated as the ratio of stroke volume (SV) to the pulmonary arterial pulse pressure (PP, i.e. difference between systolic and diastolic PAP) and expressed as $\mathrm{mL} \cdot \mathrm{mmHg}^{-1}$. The product of PVR $\left(\mathrm{mmHg} \mathrm{s} \cdot \mathrm{mL}^{-1}\right)$ and PVC $\left(\mathrm{mL} \cdot \mathrm{mmHg}^{-1}\right)$ was calculated and expressed as units of second (RC-time)

\section{Statistical analysis}

Values are expressed as the mean $\pm \mathrm{SD}$ or median (interquartile range (IQR)), unless otherwise stated. Comparisons between the age-related groups were made using a t-test or Mann-Whitney test, where appropriate. Categorical variables were compared using Chi-squared and Fisher's exact tests. Correlations between variables were calculated using Pearson's correlation coefficient or Spearman rank correlation coefficient, where appropriate. Peak exercise ULNs for RAP, mPAP, PAWP, TPG, diastolic pulmonary gradient, TPR, PVR and mPAP/CO slope were calculated as the mean $+2 \mathrm{sD} . \mathrm{p}<0.05$ was considered significant. The statistical analyses were performed using SPSS version 19 (IBM, Armonk, NY, USA).

\section{Results}

\section{Study population}

Of the 612 iCPET reports analysed, 134 subjects were found to have preserved exercise capacity $\left(V^{\prime} \mathrm{O}_{2} \max\right.$ and COmax $\geqslant 80 \%$ predicted), 66 of whom had a normal resting supine RHC (mPAP $\leqslant 20 \mathrm{mmHg}$ and PAWP $\leqslant 12 \mathrm{mmHg}$ ), yielding our study population $(\mathrm{n}=66)$. Their baseline characteristics are shown in table 1 .

The median age for the entire study population was $53(46-62)$ years. $80 \%(n=53 / 66)$ of the studied subjects were between 30 and 70 years old. Subjects $\leqslant 50$ years old constituted $38 \%(\mathrm{n}=25)$, of whom $76 \%$ $(n=19)$ were between 30 and 50 years old. Subjects aged $>50$ years constituted $62 \%(n=41)$, of whom $83 \%$ $(n=34)$ were between 51 and 70 years old. There were no differences regarding sex, body mass index, body surface area and pulmonary function testing or echocardiography measurements between the age-related groups. Older subjects had more comorbidities and pulmonary hypertension risk factors (table 1). There were no differences regarding the use of $\beta$-adrenergic blocking agents $(n=3$ for subjects $>50$ years old and $\mathrm{n}=1$ for subjects $\leqslant 50$ years old, $\mathrm{p}=0.51$ ) or diuretics $(\mathrm{n}=7$ for subjects $>50$ years old and $\mathrm{n}=2$ for subjects $\leqslant 50$ years old, $\mathrm{p}=0.26$ ) at the time of the iCPET.

\section{Resting supine haemodynamics}

At rest supine RHC, mPAP and PAWP for the entire study population were $15 \pm 2$ and $9 \pm 2 \mathrm{mmHg}$ respectively. Statistical differences between subjects aged $>50$ and $\leqslant 50$ years old were found for PP, PAWP, TPG, PVR and PVC (table 2).

\section{Noninvasive maximum incremental exercise parameters}

The median peak $V^{\prime} \mathrm{O}_{2}$ for the entire study population was $21.7(17.0-27.5) \mathrm{mL} \cdot \mathrm{kg}^{-1} \cdot \mathrm{min}^{-1}$. Peak $V^{\prime} \mathrm{O}_{2}$ as percentage of predicted was not significantly different between subjects $>50$ and $\leqslant 50$ years old (table 3 ). Older subjects presented lower peak work rate, $\mathrm{HR}$ and peak $V^{\prime} \mathrm{O}_{2}\left(\mathrm{~mL} \cdot \mathrm{kg}^{-1} \cdot \mathrm{min}^{-1}\right)$, and a higher peak systolic blood pressure. There were no significant differences regarding other noninvasive maximum exercise parameters (table 3 ).

\section{Upright haemodynamics at rest and peak exercise}

At rest upright, there were no differences for mPAP and PAWP between subjects $>50$ and $\leqslant 50$ years old. Significant differences were observed regarding CO, cardiac index (CI), SV, systemic vascular resistance (SVR), TPR, PVR and PVC (table 4). 
TABLE 1 Baseline characteristics of the study population

\begin{tabular}{|c|c|c|c|}
\hline & \multicolumn{2}{|c|}{ Age } & \multirow[t]{2}{*}{ p-value } \\
\hline & $\leqslant 50$ years & $>50$ years & \\
\hline Subjects & 25 & 41 & \\
\hline Female & $18(72)$ & $34(83)$ & 0.29 \\
\hline Age years & $44(28-47)$ & $61(54-68)$ & $<0.01$ \\
\hline BSA $\mathrm{m}^{2}$ & $1.8 \pm 0.2$ & $1.8 \pm 0.2$ & 0.73 \\
\hline $\mathrm{BMI} \mathrm{kg} \cdot \mathrm{m}^{-2}$ & $26 \pm 8$ & $27 \pm 5$ & 0.68 \\
\hline Haemoglobin $\mathrm{g} \cdot \mathrm{dL}^{-1}$ & $14.2(13.5-14.9)$ & $14.0(13.2-14.5)$ & 0.16 \\
\hline \multicolumn{4}{|l|}{ Pulmonary function testing } \\
\hline FVC \% predicted & $105 \pm 15$ & $99 \pm 15$ & 0.10 \\
\hline FEV $1 \%$ predicted & $103 \pm 13$ & $99 \pm 15$ & 0.19 \\
\hline \multicolumn{4}{|l|}{ Echocardiography ${ }^{\#}$} \\
\hline LA AP diameter mm & $33 \pm 5$ & $35 \pm 5$ & 0.27 \\
\hline LVEF \% & $60(55-65)$ & $65(60-65)$ & 0.09 \\
\hline TRV $m \cdot \mathrm{s}^{-1}$ & $2.2 \pm 0.4$ & $2.2 \pm 0.3$ & 0.72 \\
\hline Estimated sPAP mmHg & $25 \pm 6$ & $26 \pm 7$ & 0.61 \\
\hline \multicolumn{4}{|c|}{ Comorbidities and pulmonary hypertension risk factors } \\
\hline None & $20(80)$ & $21(43)$ & 0.01 \\
\hline CTD & $1(4)$ & $3(7)$ & \\
\hline Hypertension & $1(4)$ & $9(22)$ & \\
\hline OSA & $1(4)$ & $8(20)$ & \\
\hline History of PE & 2 (8) & 0 & \\
\hline
\end{tabular}

Data are presented as $n, n(\%)$, median (interquartile range) or mean $\pm S D$, unless otherwise stated. BSA: body surface area; BMI: body mass index; FVC: forced vital capacity; FEV1: forced expiratory volume in $1 \mathrm{~s}$; LA AP: left atrium anteroposterior; LVEF: left ventricular ejection fraction; TRV: tricuspid regurgitant jet velocity; SPAP: systolic pulmonary arterial pressure; CTD: connective tissue disease; OSA: obstructive sleep apnoea; PE: pulmonary embolism. \#: 45 subjects $(n=12$ subjects $\leqslant 50$ years old and $n=33$ subjects $>50$ years old) had a contemporary echocardiography report available to review; TRV could be measured in 35 subjects ( $n=7$ subjects $\leqslant 50$ years old and $n=28$ subjects $>50$ years old).

TABLE 2 Resting supine haemodynamics at right heart catheterisation

\begin{tabular}{|c|c|c|c|}
\hline & \multicolumn{2}{|c|}{ Age } & \multirow[t]{2}{*}{$p$-value } \\
\hline & $\leqslant 50$ years & $>50$ years & \\
\hline Subjects & 25 & 41 & \\
\hline RAP mmHg & $6 \pm 1$ & $5 \pm 2$ & 0.11 \\
\hline sPAP mmHg & $20 \pm 4$ & $21 \pm 4$ & 0.30 \\
\hline dPAP mmHg & $11 \pm 3$ & $10 \pm 2$ & 0.45 \\
\hline $\mathrm{PP} \mathrm{mmHg}$ & $9 \pm 3$ & $11 \pm 3$ & $<0.01$ \\
\hline mPAP mmHg & $15 \pm 3$ & $15 \pm 2$ & 0.99 \\
\hline PAWP mmHg & $10 \pm 2$ & $8 \pm 2$ & 0.01 \\
\hline TPG mmHg & $5(4-6)$ & $7(6-8)$ & $<0.01$ \\
\hline DPG mmHg & $1(0-3)$ & $2(0-3)$ & 0.45 \\
\hline $\mathrm{CO} L \cdot \min ^{-1}$ & $5.7(5.0-6.4)$ & $5.4(4.5-6.1)$ & 0.22 \\
\hline $\mathrm{CI} L \cdot \mathrm{min}^{-1} \cdot \mathrm{m}^{-2}$ & $3.1(2.8-3.3)$ & $3.0(2.6-3.4)$ & 0.38 \\
\hline SV mL & $78.1(64.1-83.4)$ & 71.1 (62.9-83.3) & 0.37 \\
\hline TPR WU & $2.68 \pm 0.65$ & $2.83 \pm 0.65$ & 0.39 \\
\hline PVR WU & $0.96 \pm 0.31$ & $1.27 \pm 0.33$ & $<0.01$ \\
\hline PVC $\mathrm{mL} \cdot \mathrm{mmHg}^{-1}$ & $8.17(6.89-11.17)$ & $6.58(5.24-7.97)$ & $<0.01$ \\
\hline RC-time s & $0.58 \pm 0.40$ & $0.48 \pm 0.12$ & 0.24 \\
\hline
\end{tabular}

Data are presented as $n$, mean \pm SD or median (interquartile range), unless otherwise stated. RAP: right atrial pressure; sPAP: systolic pulmonary arterial pressure; dPAP: diastolic pulmonary arterial pressure; PP: pulmonary arterial pulse pressure; mPAP: mean pulmonary arterial pressure; PAWP: pulmonary arterial wedge pressure; TPG: transpulmonary gradient; DPG: diastolic pulmonary gradient; CO: cardiac output; $\mathrm{Cl}$ : cardiac index; SV: stroke volume; TPR: total pulmonary resistance; PVR: pulmonary vascular resistance; WU: Wood units; PVC: pulmonary vascular compliance; RC: time constant. 
At peak exercise, mPAP was $23 \pm 5 \mathrm{mmHg}$ for the entire study population, without significant differences between the age-related groups (table 4). Peak $\mathrm{CO}$ as percentage of predicted was similar between older and younger subjects. There were differences for CO $\left(\mathrm{L} \cdot \mathrm{min}^{-1}\right), \mathrm{CI}, \mathrm{SV}, \mathrm{SVR}, \mathrm{TPR}, \mathrm{PVR}$ and PVC between subjects $>50$ and $\leqslant 50$ years old. RC-time was similar in both groups at rest and peak exercise (table 4).

TABLE 3 Noninvasive maximum incremental exercise parameters

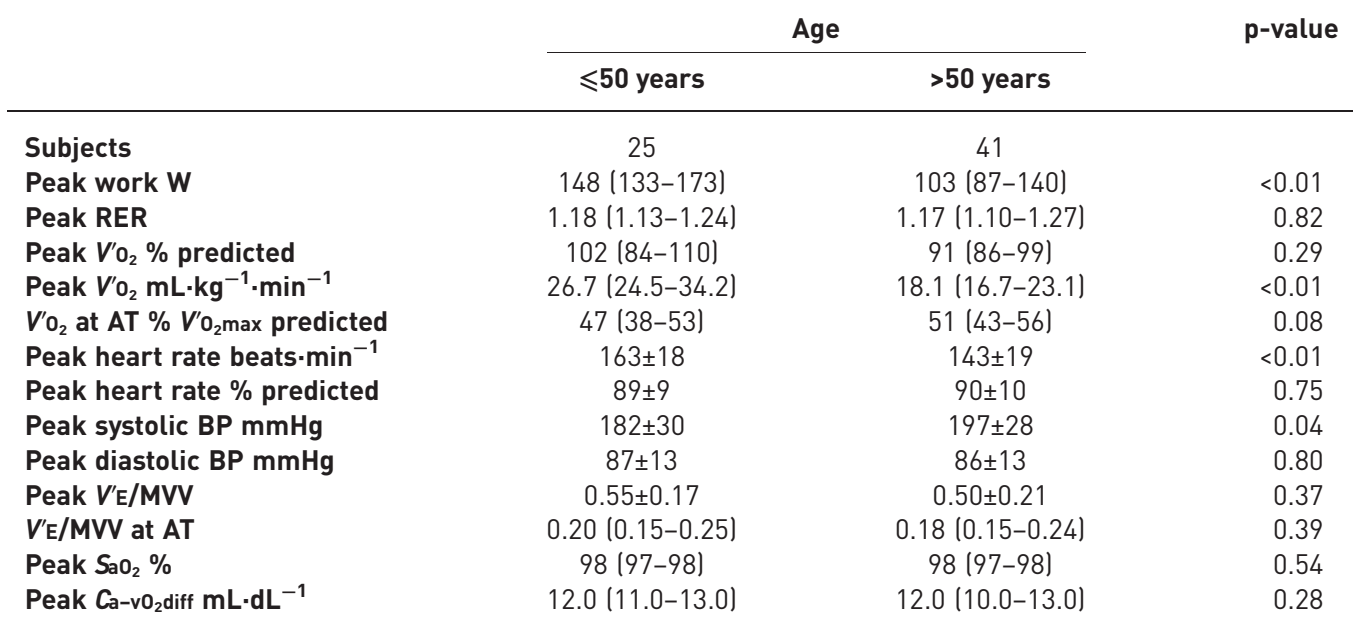

Data are presented as $\mathrm{n}$, median (interquartile range) or mean $\pm S \mathrm{D}$, unless otherwise stated. RER: respiratory exchange ratio; $V^{\prime} \mathrm{O}_{2}$ : oxygen uptake; $V^{\prime} \mathrm{O}_{2}$ max: maximal oxygen uptake; $\mathrm{AT}$ : anaerobic threshold; BP: blood pressure; $V^{\prime} E / M V V:$ ventilatory demand (minute ventilation/maximum voluntary ventilation); $\mathrm{SaO}_{2}$ : arterial oxygen saturation; $\mathrm{Ca}_{\mathrm{a}} \mathrm{vO}_{2}$ diff: arterial-mixed venous oxygen content difference.

TABLE 4 Upright haemodynamics at rest and peak exercise

\begin{tabular}{|c|c|c|c|c|}
\hline & \multicolumn{4}{|c|}{ Age } \\
\hline & \multicolumn{2}{|c|}{$\leqslant 50$ years } & \multicolumn{2}{|c|}{$>50$ years } \\
\hline & Rest & Peak & Rest & Peak \\
\hline Subjects & \multicolumn{2}{|c|}{25} & \multicolumn{2}{|c|}{41} \\
\hline RAP $\mathrm{mmHg}$ & $2 \pm 1$ & $5 \pm 4$ & $2 \pm 1$ & $5 \pm 4$ \\
\hline sPAP mmHg & $17 \pm 4$ & $33 \pm 7$ & $18 \pm 4$ & $36 \pm 8$ \\
\hline dPAP $\mathrm{mmHg}$ & $8 \pm 4$ & $15 \pm 5$ & $8 \pm 3$ & $15 \pm 5$ \\
\hline $\mathrm{PP} \mathrm{mmHg}$ & $9 \pm 2$ & $19 \pm 5$ & $9 \pm 3$ & $20 \pm 6$ \\
\hline mPAP $\mathrm{mmHg}$ & $12 \pm 4$ & $22 \pm 4$ & $12 \pm 3$ & $23 \pm 5$ \\
\hline PAWP mmHg & $4 \pm 3$ & $9 \pm 5$ & $4 \pm 3$ & $9 \pm 4$ \\
\hline TPG mmHg & $8(6-9)$ & $12(10-16)$ & $8(7-9)$ & $14(11-17)$ \\
\hline DPG mmHg & $3(2-5)$ & $6(2-9)$ & $4(3-5)$ & $6(3-8)$ \\
\hline $\mathrm{CO} L \cdot \mathrm{min}^{-1}$ & $5.9(4.6-7.8)$ & $16.2(13.8-19.2)$ & $5.0(3.8-5.8)^{\#}$ & $12.1(9.4-14.2)^{\pi}$ \\
\hline Peak CO $\%$ predicted & & $116(97-125)$ & & $112(101-126)$ \\
\hline $\mathrm{Cl} \mathrm{L} \cdot \mathrm{min}^{-1} \cdot \mathrm{m}^{-2}$ & $3.2(2.7-3.7)$ & $8.6(8.0-10.3)$ & $2.6(2.2-3.1)^{+}$ & $6.6(5.9-7.9)^{\pi}$ \\
\hline SV mL & $70.0(61.0-93.0)$ & 102.7 (80.7-113.5) & $54.0(42.0-73.5)^{+}$ & $81.9(69.4-97.8)^{+}$ \\
\hline SVR WU & $17.24 \pm 5.04$ & $7.22 \pm 1.78$ & $21.71 \pm 5.66^{\#}$ & $10.08 \pm 2.74^{+}$ \\
\hline TPR WU & $2.07 \pm 0.82$ & $1.39 \pm 0.36$ & $2.63 \pm 1.09^{\#}$ & $1.98 \pm 0.59$ ? \\
\hline PVR WU & $1.31 \pm 0.53$ & $0.82 \pm 0.26$ & $1.68 \pm 0.57^{\#}$ & $1.20 \pm 0.45^{\natural}$ \\
\hline PVC $\mathrm{mL} \cdot \mathrm{mmHg}^{-1}$ & 8.70 (6.85-11.55) & $5.71(4.61-6.88)$ & $6.50(4.70-8.90)^{+}$ & $4.10(3.41-6.02)^{+}$ \\
\hline RC-time s & $0.68 \pm 0.25$ & $0.28 \pm 0.11$ & $0.66 \pm 0.22$ & $0.30 \pm 0.07$ \\
\hline
\end{tabular}

Data are presented as $n$, mean $\pm S D$ or median (interquartile range). RAP: right atrial pressure; sPAP: systolic pulmonary arterial pressure; dPAP: diastolic pulmonary arterial pressure; PP: pulmonary arterial pulse pressure; mPAP: mean pulmonary arterial pressure; PAWP: pulmonary arterial wedge pressure; TPG: transpulmonary gradient; DPG: diastolic pulmonary gradient; $\mathrm{CO}$ : cardiac output; $\mathrm{Cl}$ : cardiac index; SV: stroke volume; SVR: systemic vascular resistance; TPR: total pulmonary resistance; PVR: pulmonary vascular resistance; WU: Wood units; PVC: pulmonary vascular compliance; RC: time constant. ${ }^{\#}$ : $p<0.05$ comparing subjects $>50$ and $\leqslant 50$ years; " : $p<0.001$ comparing subjects $>50$ and $\leqslant 50$ years; ${ }^{+}: p<0.01$ comparing subjects $>50$ and $\leqslant 50$ years. 
There were significant inverse correlations between age and $\mathrm{CO}(\mathrm{r}=-0.60 ; \mathrm{p}<0.001)$ and $\mathrm{CI}(\mathrm{r}=-0.70$; $\mathrm{p}<0.001$ ), and a positive correlation between age and PVR $(\mathrm{r}=0.56 ; \mathrm{p}<0.001)$ (figure 1$)$. mPAP/CO slopes were higher in older subjects (figure 2).

ULNs for maximum upright exercise pulmonary haemodynamics are presented in table 5 and figure 3 .

\section{Discussion}

In this study of subjects with preserved exercise capacity and normal resting supine haemodynamics who underwent a maximum incremental cycling exercise protocol, we found that subjects aged $>50$ and $\leqslant 50$ years old have different pulmonary vascular responses to exercise. Older subjects have higher PVR, TPR and lower PVC at peak exercise compared with the younger population.

The current study included only subjects with preserved exercise capacity [17] determined by a normal $V^{\prime} \mathrm{O}_{2} \max$ and COmax, effectively excluding subjects with any clinically relevant "silent pathology", such as exercise pulmonary hypertension, that would be otherwise uncovered during exercise [4, 23, 24]. Additionally, and in contrast to prior reports, all subjects performed the same standardised maximum incremental cycling exercise protocol with a consistent and validated method of haemodynamic waveform analysis [20], the latter of which avoids the overestimation of pulmonary vascular pressures [25]. Limited data has been published to date for normal subjects $>50$ years old at maximum exercise. Our study includes 41 subjects $>50$ years of age and therefore provides an interesting insight for this population.

\section{Resting supine haemodynamics}

At resting supine RHC, we found differences between subjects $>50$ and $\leqslant 50$ years old for PP, PAWP, TPG, PVR and PVC, but not for TPR, CO or CI. Although within the normal range and likely not clinically relevant, the lower PAWP in older subjects led to a higher TPG and a higher PVR at the same CO. In a similar manner, the higher PP in older subjects was observed with a lower PVC at the same SV. As a result, RC-time was not significantly different between the age-related groups, reflecting the hyperbolic relationship between PVR and PVC [26].

These observations suggest that when supine and at rest, age-related differences might be related to intrinsic properties of the pulmonary vasculature. Ageing is known to be associated with functional, structural and mechanical changes of the systemic vasculature [27]. Therefore, age-related microvascular changes such as chronic oxidative stress, inflammation, endothelial dysfunction, increased vascular stiffness and progressive vascular remodelling [27-29] may in part explain our resting supine haemodynamic findings.

Despite the fact that no differences were found for diuretic use between groups, the presence of mild fluid deficit in the older population is a possibility that we cannot entirely exclude. However, at rest upright PAWP was $4 \pm 3 \mathrm{mmHg}$ for both groups, indicating that subjects $>50$ and $\leqslant 50$ years old had the same overall "fluid status" in the upright position.

\section{MPAP and PAWP at peak exercise}

We found that peak exercise mPAP and PAWP were not significantly different between subjects $>50$ and $\leqslant 50$ years old. The ULN for peak mPAP found in our study was $33 \mathrm{mmHg}$ for older subjects and
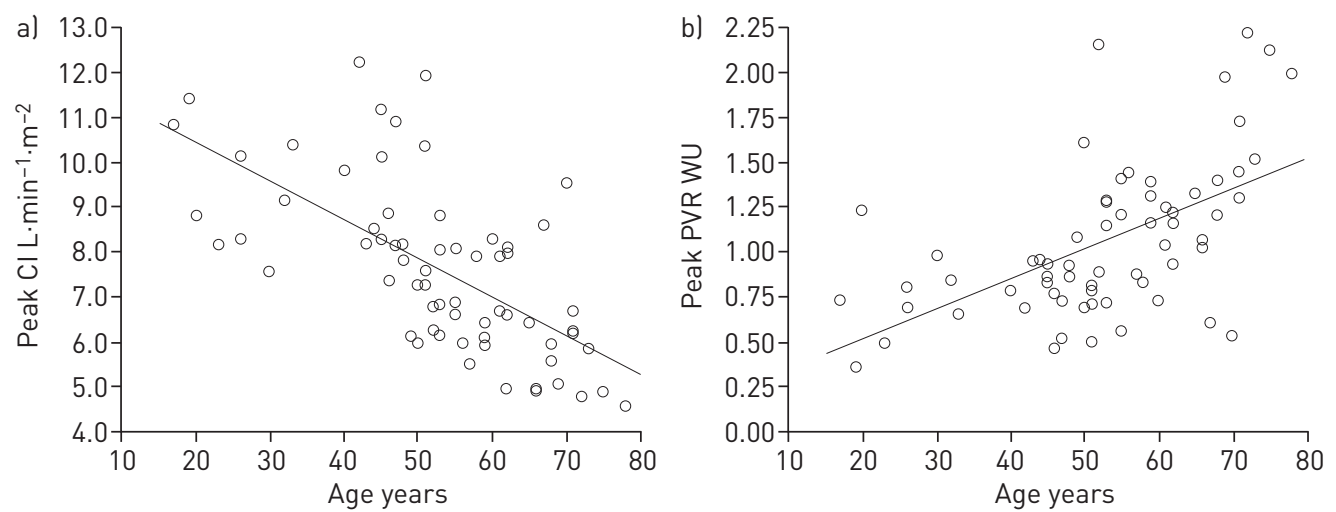

FIGURE 1 Correlation between age and a) peak cardiac index ( $\mathrm{Cl}$ ( $\mathrm{r}=-0.70$; $\mathrm{p}<0.001)$ and b) peak pulmonary vascular resistance (PVR) ( $r=0.56 ; p<0.001)$. WU: Wood units. 
FIGURE 2 Mean pulmonary arterial pressure relative to cardiac output (mPAP/CO slope) from rest upright to peak upright exercise. Data represent the mean \pm SD (error bars). ${ }^{\#}$ : $p<0.001$ comparing subjects $>50$ and $\leqslant 50$ years old.

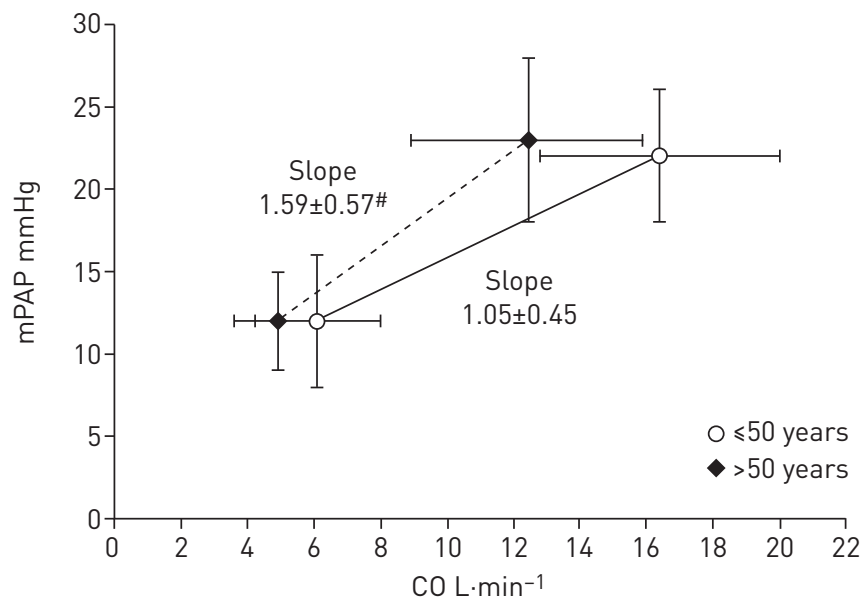

$30 \mathrm{mmHg}$ for younger subjects. Peak PAWP ULN was 17 and $19 \mathrm{mmHg}$ for older and younger subjects respectively.

Our results differ from previous reports that identified higher ULN for mPAP during exercise for subjects $>50$ years old [12]. Kovacs et al. [12], in a systematic review of the available literature, found that nearly half of subjects $>50$ years old developed a mPAP $>30 \mathrm{mmHg}$ during slight exercise. Peak PAWP was $15 \pm 8 \mathrm{mmHg}$ for maximal upright exercise irrespective of age, with an ULN of $31 \mathrm{mmHg}$ [12]. During slight supine exercise, peak PAWP for subjects $>50$ years old was $17 \pm 6.5 \mathrm{mmHg}$, with an ULN of $30 \mathrm{mmHg}$ [12]. However, these peak PAWP ULNs are higher than the previous published cut-off peak PAWP value of $20 \mathrm{mmHg}$ [30-32] and therefore might be indicative of the inclusion of subjects with occult left heart diastolic dysfunction (exercise pulmonary venous hypertension), as appropriately acknowledged by the authors [12]. As a result, the higher peak PAWP might have ultimately influenced peak mPAP, leading to an elevated exercise mPAP ULN for subjects $>50$ years old.

Recent studies have described the contribution of elevated left ventricular filling pressures and age-related diastolic dysfunction on peak exercise mPAP values in subjects $>55$ years old [15]. VAN EMPEL et al. [15] found a peak supine PAWP of $14 \pm 4.4$ for subjects $<55$ years old and $18 \pm 6.4$ for subjects $>55$ years old that, similar to Kovacs et al. [12], would lead to an exercise PAWP ULN of $31 \mathrm{mmHg}$. However, whether the reported peak PAWP reflected a physiologic or pathologic response to exercise is uncertain.

The absence of concomitant metabolic measurements in previous reports (i.e. $V^{\prime} \mathrm{O}_{2} \max$ ) might have allowed the inclusion of subjects with reduced exercise capacity and therefore possible pathologic pulmonary vascular responses to exercise. Exercise pulmonary venous hypertension is known to have an associated reduced aerobic capacity, which is related to a blunted CO response during exercise [23]. Therefore, the

TABLE 5 Upper limits of normal for maximum upright exercise pulmonary haemodynamics

\begin{tabular}{lcc} 
& & Age \\
\cline { 2 - 3 } & $\leqslant \mathbf{5 0}$ years & $\mathbf{5 0}$ years \\
\hline Subjects $\mathbf{n}$ & 25 & 41 \\
RAP $\mathbf{m m H}$ & 13 & 13 \\
mPAP $\mathbf{m m g}$ & 30 & 33 \\
PAWP $\mathbf{~ m H g}$ & 19 & 17 \\
TPG $\mathbf{m m g}$ & 21 & 22 \\
DPG $\mathbf{m m H g}$ & 14 & 12 \\
TPR WU & 2.11 & $3.16^{\#}$ \\
PVR WU & 1.34 & $2.10^{\#}$ \\
mPAP/CO slope & 1.95 & $2.73^{\#}$
\end{tabular}

Data represent the mean+2SD, unless otherwise stated. RAP: right atrial pressure; mPAP: mean pulmonary arterial pressure; PAWP: pulmonary arterial wedge pressure; TPG: transpulmonary gradient; DPG: diastolic pulmonary gradient; TPR: total pulmonary resistance; PVR: pulmonary vascular resistance; WU: Wood units; CO: cardiac output. " : $p<0.001$ comparing subjects $>50$ and $\leqslant 50$ years. 


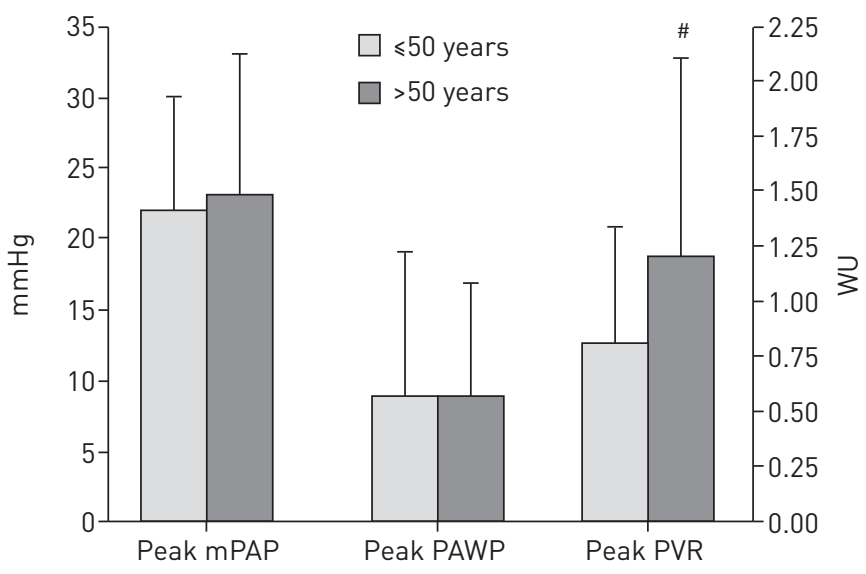

FIGURE 3 Mean pulmonary arterial pressure (mPAP), pulmonary arterial wedge pressure (PAWP) and pulmonary vascular resistance (PVR) upper limits of normal for maximum upright exercise. Data are presented as mean+2sD (error bars). \#: $p<0.001$ comparing subjects $>50$ and $\leqslant 50$ years old. WU: Wood units.

requirement of a preserved $V^{\prime} \mathrm{O}_{2} \max$ and COmax in our study likely excluded subjects with clinically relevant exercise-induced pulmonary venous hypertension, in which peak mPAP ULN would be affected by pathologic elevations of the PAWP during exercise.

\section{TPR, PVR and PVC at peak exercise}

We found that PVR and TPR at peak exercise were significantly higher in subjects $>50$ years old. Peak exercise PVR ULNs were $2.10 \mathrm{WU}$ for subjects $>50$ years old and $1.34 \mathrm{WU}$ for subjects $\leqslant 50$ years old. Peak TPR ULN were 3.16 and 2.11 WU for older and younger subjects, respectively. Additionally, we observed a positive correlation between peak PVR and age, and a negative correlation between CI and age (figure 1). Our results are similar to previous reports showing decreased peak $\mathrm{CO}[28,33]$ and higher peak PVR for the older population $[13,15]$. Moreover, $\mathrm{mPAP} / \mathrm{CO}$ slopes were higher in older subjects (figure 2).

In addition to the lower $\mathrm{CO}$ at peak exercise in subjects $>50$ years old, the lower PVC at rest and peak exercise in these subjects suggest intrinsic pulmonary vascular changes as a function of normal ageing [28], ultimately indicating a lower pulmonary vascular reserve in older subjects. This observation is more pronounced during exercise, when pulmonary vessels become less compliant (stiffer) as their diameters increase due to increased flow [34]. Another mechanism that could explain the decrease in PVC and its differences between the age-related groups is the exercise-induced sympathetic nervous system stimulation during exercise [35], which could be more pronounced in older subjects [36]. However, in an analogous manner to PVR and CO, the age-related decrease in peak PVC was accompanied by a decreased peak SV.

In established resting pulmonary arterial hypertension, pulmonary vascular remodelling is thought in most cases to lead to right ventricular dysfunction (and a low CO) [37] through increased right heart afterload. Little is known, however, about right ventricular/pulmonary vascular coupling across ages in normal subjects. It is uncertain whether age-related changes in the pulmonary vasculature lead to a decrease in peak CO or if intrinsic changes of the senescent heart decrease exercise CO and SV in a primary manner leading to secondary changes in PVR and PVC. Therefore, we speculate that in addition to age-related pulmonary vascular changes in structure and function, the senescent heart and its blunted CO and SV response during exercise might influence in part exercise pulmonary haemodynamics as a function of normal ageing. However, because the present study by design is observational, further work will be necessary to better define any age-related causal relationship between intrinsic cardiac function and the pulmonary vascular response to exercise.

As a result of the observed decrease in PVR and PVC at maximum exercise, RC-time decreased by almost half without significant differences between subjects $>50$ and $\leqslant 50$ years old. This RC-time exercise pattern in healthy subjects has been recently reported by others [38], and might be indicative of the relative increase in pulsatile load and consequent pulmonary vascular bed recruitment during exercise [38].

\section{Potential implications of the current study}

Uncertainty concerning the ULNs for exercise haemodynamics in subjects $>50$ years of age $[2,3]$ contributed in part to the decision to withdraw the exercise criteria from the pulmonary hypertension guidelines in 2008 [2]. Therefore, the identification of age-related maximum exercise haemodynamic patterns in the current study could assist the differentiation between a physiologic and a pathologic pulmonary vascular response to exercise, contributing to delineate exercise pulmonary hypertension diagnostic criteria and providing an additional framework for the future understanding of exercise pulmonary hypertension natural history. 
An important finding of the current study is the identification of peak PAWP ULN $<20 \mathrm{mmHg}$, also for subjects $>50$ years of age. Additionally, peak mPAP was similar between the age-related groups. However, the overall pulmonary vascular response to exercise was different between older and younger subjects. These findings corroborate the knowledge that proper interpretation of exercise haemodynamics must rely on other variables in addition to peak mPAP, such as peak PVR, peak TPR or mPAP/CO slope $[4,22,39,40]$.

We identified a TPR ULN of $2.11 \mathrm{WU}$ and mPAP/CO slope ULN of $1.95 \mathrm{WU}$ for subjects $\leqslant 50$ years old (table 5), a value that is lower than the proposed cut-off of $3.0 \mathrm{WU}[22,39,40]$. Therefore, the present data might favour the idea that identification of pathologic pulmonary vascular responses to exercise would benefit from the use of age-related cut-off values. However, the implications of our results must be carefully considered, since they were derived from a single-centre study. Moreover, age-related changes in pulmonary vascular response to exercise are likely to be a continuum across ages and the use of any age cut-off value should be interpreted with caution.

\section{Limitations}

Our study population was derived from the iCPET evaluation of subjects with unexplained exertional intolerance and they may not represent a completely healthy population. However, studied subjects were selected based on a preserved exercise capacity defined by objective criteria $\left(V^{\prime} \mathrm{O}_{2} \max\right.$ and $\left.\mathrm{COmax}\right)$. Therefore, they constitute a population with normal (physiologic) responses to exercise and represent "symptomatic normal" subjects. Additionally, despite the existence of comorbidities, we effectively excluded the presence of clinically relevant pulmonary vascular disease and exercise pulmonary venous hypertension (which are associated with a reduced exercise capacity) by the aforementioned inclusion criteria. Finally, large-scale prospective iCPET studies in asymptomatic healthy subjects are unlikely to be undertaken at the current time. Haemodynamic data for subjects with connective tissue disease or history of pulmonary embolism $(\mathrm{n}=6)$ is provided in online supplementary table S1.

The age cut-off value of 50 years in our study was selected due to the uncertainty of the exercise haemodynamics ULNs for subjects $>50$ years old, as pointed out in the Fourth and Fifth World Symposia on Pulmonary Hypertension [2,3], and might be considered an arbitrary value. Our results obtained using this age cut-off value were equivalent to the results obtained using the median age of our sample (53 years) to determine the age-related groups (data not shown). However, in the present study most of the subjects were $\leqslant 70$ years old and therefore we have limited ability to comment on exercise haemodynamics ULN for subjects $>70$ years old (haemodynamic data for subjects $>70$ years old is provided in online supplementary table S2). Additionally, differences in pulmonary vascular response to exercise are probably a continuum as a function of normal ageing and therefore ULNs derived from any age-related cut-off value have to be interpreted with caution.

It is possible that trained, but symptomatic subjects were included in our study. These subjects can have compliant atria and ventricles as part of the remodelled athletic heart yielding low filling pressures while exercising, which could influence in part our results. However, all studied subjects met our inclusion criteria of normal $V^{\prime} \mathrm{O}_{2} \max$, COmax and RHC, representing therefore a spectrum of normal subjects. Finally, we calculated resting supine $\mathrm{CO}$ by the Fick method using an estimated $V^{\prime} \mathrm{O}_{2}$; consequently, resting supine CO, CI, PVR and PVC results should be interpreted with caution. However, our RHC inclusion criteria used only pressures, so any such error did not affect inclusion criteria or peak exercise results.

\section{Conclusions}

In this study of subjects with preserved exercise capacity and normal resting supine haemodynamics, we demonstrated that subjects $>50$ and $\leqslant 50$ years old have different pulmonary vascular responses to exercise, identified by a higher peak PVR and peak TPR, and a lower peak PVC, in subjects $>50$ years old, resulting in different exercise haemodynamics ULN compared with the younger population. Further studies are necessary to determine the exact mechanisms underlying the age-related changes in exercise haemodynamics and to distinguish normal from pathological ageing.

\section{Acknowledgements}

We thank David Yang (Division of Nuclear Medicine and Cardiovascular Imaging, Department of Radiology, Brigham and Women's Hospital, Harvard Medical School, Boston, MA, USA) for his technical expertise.

\section{References}

1 Rich S, Dantzker DR, Ayres SM, et al. Primary pulmonary hypertension. A national prospective study. Ann Intern Med 1987; 107: 216-223.

2 Badesch DB, Champion HC, Sanchez MAG, et al. Diagnosis and assessment of pulmonary arterial hypertension. J Am Coll Cardiol 2009; 54: S55-S66.

3 Hoeper MM, Bogaard HJ, Condliffe R, et al. Definitions and diagnosis of pulmonary hypertension. J Am Coll Cardiol 2013; 62: D42-D50. 
Tolle JJ, Waxman AB, Van Horn TL, et al. Exercise-induced pulmonary arterial hypertension. Circulation 2008; 118: 2183-2189.

5 Fowler RM, Maiorana AJ, Jenkins SC, et al. Implications of exercise-induced pulmonary arterial hypertension. Med Sci Sports Exerc 2011; 43: 983-989.

6 Kovacs G, Maier R, Aberer E, et al. Borderline pulmonary arterial pressure is associated with decreased exercise capacity in scleroderma. Am J Respir Crit Care Med 2009; 180: 881-886.

7 Saggar R, Khanna D, Shapiro S, et al. Brief report: effect of ambrisentan treatment on exercise-induced pulmonary hypertension in systemic sclerosis: a prospective single-center, open-label pilot study. Arthritis Rheum 2012; 64: 4072-4077.

8 Steen V, Chou M, Shanmugam V, et al. Exercise-induced pulmonary arterial hypertension in patients with systemic sclerosis. Chest 2008; 134: 146-151.

9 Condliffe R, Kiely DG, Peacock AJ, et al. Connective tissue disease-associated pulmonary arterial hypertension in the modern treatment era. Am J Respir Crit Care Med 2009; 179: 151-157.

10 Galiè N, Humbert M, Vachiery J-L, et al. 2015 ESC/ERS Guidelines for the diagnosis and treatment of pulmonary hypertension. Eur Respir J 2015; 46: 903-975.

11 Granath A, Jonsson B, Strandell T. Circulation in healthy old men, studied by right heart catheterization at rest and during exercise in supine and sitting position. Acta Med Scand 1964; 176: 425-446.

12 Kovacs G, Berghold A, Scheidl S, et al. Pulmonary arterial pressure during rest and exercise in healthy subjects: a systematic review. Eur Respir J 2009; 34: 888-894.

13 Kovacs G, Olschewski A, Berghold A, et al. Pulmonary vascular resistances during exercise in normal subjects: a systematic review. Eur Respir J 2012; 39: 319-328.

14 Argiento P, Vanderpool RR, Mulè M, et al. Exercise stress echocardiography of the pulmonary circulation: limits of normal and sex differences. Chest 2012; 142: 1158-1165.

15 van Empel VPM, Kaye DM, Borlaug BA. Effects of healthy aging on the cardiopulmonary hemodynamic response to exercise. Am J Cardiol 2014; 114: 131-135.

16 Maron BA, Cockrill BA, Waxman AB, et al. The invasive cardiopulmonary exercise test. Circulation 2013; 127: 1157-1164.

17 Hansen JE, Sue DY, Wasserman K. Predicted values for clinical exercise testing. Am Rev Respir Dis 1984; 129: S49-S55.

18 Wasserman K, Hansen JE, Sue DY, et al. Principles of exercise testing and interpretation. 4th Edn. Philadelphia, Lippincott Williams \& Wilkins, 2005.

19 Kovacs G, Avian A, Olschewski A, et al. Zero reference level for right heart catheterization. Eur Respir J 2013; 42: 1586-1594.

20 Boerrigter BG, Waxman $\mathrm{AB}$, Westerhof $\mathrm{N}$, et al. Measuring central pulmonary pressures during exercise in COPD: how to cope with respiratory effects? Eur Respir J 2013; 43: 1316-1325.

21 Beck KC, Randolph LN, Bailey KR, et al. Relationship between cardiac output and oxygen consumption during upright cycle exercise in healthy humans. J Appl Physiol 2006; 101: 1474-1480.

22 Lewis GD, Bossone E, Naeije R, et al. Pulmonary vascular hemodynamic response to exercise in cardiopulmonary diseases. Circulation 2013; 128: 1470-1479.

23 Santos M, Opotowsky AR, Shah AM, et al. Central cardiac limit to aerobic capacity in patients with exertional pulmonary venous hypertension: implications for heart failure with preserved ejection fraction. Circ Heart Fail 2015; 8: 278-285.

24 Naeije R, Vonk Noordegraaf A, Kovacs G. Exercise-induced pulmonary hypertension: at last! Eur Respir J 2015; 46: $583-586$.

25 Kovacs G, Avian A, Pienn M, et al. Reading pulmonary vascular pressure tracings. How to handle the problems of zero leveling and respiratory swings. Am J Respir Crit Care Med 2014; 190: 252-257.

26 Saouti N, Westerhof N, Postmus PE, et al. The arterial load in pulmonary hypertension. Eur Respir Rev 2010; 19: $197-203$.

27 Harvey A, Montezano AC, Touyz RM. Vascular biology of ageing - implications in hypertension. J Mol Cell Cardiol 2015; 83: 112-121.

28 Lakatta EG, Mitchell JH, Pomerance A, et al. Human aging: changes in structure and function. J Am Coll Cardiol 1987; 10: 42A-47A

29 Sawabe M. Vascular aging: from molecular mechanism to clinical significance. Geriatr Gerontol Int 2010; 10: Suppl. 1, S213-S220.

30 Groves BM, Reeves JT, Sutton JR, et al. Operation Everest II: elevated high-altitude pulmonary resistance unresponsive to oxygen. J Appl Physiol 1987; 63: 521-530.

31 Wagner PD, Gale GE, Moon RE, et al. Pulmonary gas exchange in humans exercising at sea level and simulated altitude. J Appl Physiol 1986; 61: 260-270.

32 Parker JO, Thadani U. Cardiac performance at rest and during exercise in normal subjects. Bull Eur Physiopathol Respir 1979; 15: 935-949.

33 Brandfonbrener M, Landowne M, Shock NW. Changes in cardiac output with age. Circulation 1955; 12: 557-566.

34 Elkins RC, Milnor WR. Pulmonary vascular response to exercise in the dog. Circ Res 1971; 29: 591-599.

35 Naeije R, Chesler N. Pulmonary circulation at exercise. Compr Physiol 2012; 2: 711-741.

36 Sun Z. Aging, arterial stiffness, and hypertension. Hypertension 2015; 65: 252-256.

37 Vonk-Noordegraaf A, Haddad F, Chin KM, et al. Right heart adaptation to pulmonary arterial hypertension: physiology and pathobiology. J Am Coll Cardiol 2013; 62: D22-D33.

38 Claessen G, La Gerche A, Dymarkowski S, et al. Pulmonary vascular and right ventricular reserve in patients with normalized resting hemodynamics after pulmonary endarterectomy. J Am Heart Assoc 2015; 4: e001602.

39 Naeije R, Vanderpool R, Dhakal BP, et al. Exercise-induced pulmonary hypertension: physiological basis and methodological concerns. Am J Respir Crit Care Med 2013; 187: 576-583.

40 Herve P, Lau EM, Sitbon O, et al. Criteria for diagnosis of exercise pulmonary hypertension. Eur Respir J 2015; 46: 728-737. 